\title{
Measurements of copper and cesium telluride cathodes in a radio-frequency photoinjector
}

\author{
Eduard Prat, Simona Bettoni, Hans-Heinrich Braun, Romain Ganter, and Thomas Schietinger \\ Paul Scherrer Institut, CH-5232 Villigen PSI, Switzerland
}

(Received 11 November 2014; published 9 April 2015)

\begin{abstract}
Radio-frequency (rf) photoinjectors are commonly used to generate intense bright electron beams for a wide range of applications, most notably as drivers for X-ray Free-Electron Lasers. The photocathode, mounted inside an rf gun and illuminated by a suitable laser, thereby plays a crucial role as the source of the electrons. The intrinsic emittance and the quantum efficiency of the electron source are determined by the properties of the photocathode's surface material. We present measurements of the intrinsic emittance and the quantum efficiency performed with copper and cesium telluride cathodes in the same rf photoinjector, thus comparing, for the first time, the performance of metal and semiconductor cathodes under the same conditions. Our results are consistent with theoretical expectations and show that the difference in intrinsic emittance for the two types of material is not significant in view of accelerator applications. We conclude that cesium telluride photocathodes provide a much higher quantum efficiency at essentially negligible degradation in beam emittance.
\end{abstract}

DOI: 10.1103/PhysRevSTAB.18.043401

\section{INTRODUCTION}

Radio-frequency (rf) photoinjectors [1] are the most effective way to generate intense bright electron beams. Electrons are produced via the photoelectric effect by a suitable laser impinging on a photocathode mounted inside an rf gun. The rf field of the gun is synchronized to accelerate the electrons to an energy of up to several $\mathrm{MeV}$. $\mathrm{Rf}$ photoinjectors find a range of applications, for instance in compact gamma-ray or X-ray sources based on inverse Compton scattering [2,3], or as direct electron sources for ultrafast time-resolved pump-probe electron diffraction $[4,5]$. By far the strongest interest in rf photoinjectors arises from their use as drivers for high-brightness electron injectors for future linear colliders or X-ray Free-Electron Laser (FEL) facilities, where, in addition to high brightness, a well-defined pulse structure is required. X-ray FELs are cutting-edge research tools for various fields of science such as biology, physics, chemistry, medicine, and material science. Most of the present and planned X-ray FELs employ laser-driven rf photoinjectors as electron beam sources, including SwissFEL under construction at the Paul Scherrer Institute [6].

The photocathode material in an rf photoinjector is of vital importance since it determines the intrinsic emittance and the quantum efficiency $(\mathrm{QE})$ of the electron source. Semiconductor materials like cesium telluride

\footnotetext{
*eduard.prat@psi.ch
}

Published by the American Physical Society under the terms of the Creative Commons Attribution 3.0 License. Further distribution of this work must maintain attribution to the author $(s)$ and the published article's title, journal citation, and DOI.
PACS numbers: 29.25.Bx, 41.60.Cr, 52.59.Wd, 85.60.Ha

$\left(\mathrm{Cs}_{2} \mathrm{Te}\right)$ provide a $\mathrm{QE}$ orders of magnitude larger than metal photocathodes like copper $(\mathrm{Cu})$, while the expected intrinsic emittance for both types of material is similar. A higher QE implies a more affordable laser system and allows utilizing the potential excess of laser power to optimize the transverse and longitudinal shape of the laser pulses, thereby reducing the final emittance of the electron beam $[7,8]$.

Extensive worldwide research and development has been performed in the last years to measure the intrinsic emittance of metals and semiconductors in rf photoinjectors, see for example Refs. [9-11]. In general these earlier studies have obtained emittance values in the upper range or above of what is expected from theory. Furthermore the performance of metals and semiconductors as electron sources have so far never been compared systematically in the exact same environment. Only in this case both the accelerator conditions (including rf and laser) and the measurement procedure are equal, thus allowing a clean comparison. This is an important consideration in view of the fact that emittance values between different facilities or labs are notoriously difficult to compare.

We have measured the performance of $\mathrm{Cu}$ and $\mathrm{Cs}_{2} \mathrm{Te}$ under the same conditions at the SwissFEL Injector Test Facility [12]. This $250 \mathrm{MeV}$ accelerator based on an $S$-band $(3 \mathrm{GHz}) \mathrm{rf}$ photoinjector was operated between 2010 and 2014 with the principal goal of demonstrating the highbrightness electron beam required for SwissFEL [6]. We have found that the difference in intrinsic emittance between $\mathrm{Cu}$ and $\mathrm{Cs}_{2} \mathrm{Te}$ is minimal. In contrast to some previous research, our results are consistent with theoretical expectations. We attribute this advance to improvements in the emittance measurement method: we have developed an accurate high-resolution method to measure the emittance 
of very low charge beams ( $1 \mathrm{pC}$ and less), which are not affected by space-charge effects, a necessary condition to measure the intrinsic emittance [13].

The intrinsic emittance is proportional to the transverse size of the photo-emitted beam and is related to the initial kinetic energy or effective temperature of the electrons leaving the cathode surface. It has a strong impact on the final emittance of the electron beam-for instance, numerical simulations done for SwissFEL for a bunch charge of $200 \mathrm{pC}$ show that the contribution of the intrinsic emittance to the final emittance is around $70 \%$ for a fully optimized linac [14].

The electron beam emittance is of fundamental relevance for FEL machines. First, transversely coherent radiation is generated only if $\varepsilon_{n} / \gamma \approx \lambda / 4 \pi$, where $\varepsilon_{n}$ is the normalized beam emittance, $\gamma$ is the Lorentz factor and $\lambda$ is the FEL radiation wavelength. This condition implies that by reducing the normalized emittance the final beam energy can be decreased, which translates into a shorter and more affordable accelerator. Second, for a given beam energy, a smaller emittance implies a higher radiation power and a shorter undulator beamline to reach FEL saturation. For instance, for the SwissFEL case, the FEL power increases about threefold if the emittance is reduced from the nominal value of $0.43 \mu \mathrm{m}$ to $0.25 \mu \mathrm{m}$ [13].

The intrinsic emittance for both metal and semiconductor photocathodes can be expressed as [15-17]

$$
\varepsilon_{\mathrm{int}}=\sigma_{l} \sqrt{\frac{2 E_{K}}{3 m_{e} c^{2}}},
$$

where $\sigma_{l}$ is the RMS laser beam size and $m_{e} c^{2}$ the electron's rest mass energy. We call $\varepsilon_{\text {int }} / \sigma_{l}$ the normalized intrinsic emittance, expressed in $\mu \mathrm{m} / \mathrm{mm}$, as it is independent of the laser beam size. The average kinetic energy of the photoemitted electrons at the cathode surface, denoted by $E_{K}$, is expressed differently for metal and semiconductors:

$$
\begin{gathered}
\text { metals: } 2 E_{K}=\phi_{l}-\phi_{w}+\phi_{\mathrm{Sch}}, \\
\text { semiconductors: } 2 E_{K}=\phi_{l}-E_{g}-E_{a}+\phi_{\mathrm{Sch}},
\end{gathered}
$$

where $\phi_{l}$ is the laser photon energy, $\phi_{w}$ is the work function of the material, $E_{g}$ is the gap energy, $E_{a}$ is the electron affinity, and $\phi_{\text {Sch }}$ is due to the Schottky effect, which takes into account the reduction of the barrier in the presence of an applied electric field. The Schottky contribution to the intrinsic emittance is expressed as [18]:

$$
\phi_{\mathrm{Sch}}=\sqrt{\frac{e^{3}}{4 \pi \varepsilon_{0}} \beta E_{c}},
$$

where $e$ is the charge of the electron, $\varepsilon_{0}$ is the vacuum permittivity, $\beta$ is the local field enhancement factor, which includes effects related to the cathode surface properties, and $E_{c}$ is the applied field on the cathode.

The work function of copper, the electron affinity of cesium telluride, and the local field enhancement factor $\beta$ are very sensitive to cathode surface properties. There is a large range of reported work functions for copper in the literature: from the different published values, the average work function is estimated to be $4.66 \pm 0.51 \mathrm{eV}$ [19]. The reported values of the threshold energy $\left(E_{g}+E_{a}\right)$ for cesium telluride vary between $3.5 \mathrm{eV}$ and $4.6 \mathrm{eV}$ [20-22]. Finally, published values of the local field enhancement factor $\beta$ range between 1 and 5 or higher $[9,23]$. For a $\beta$ between 1 and 5 , the Schottky effect contribution, assuming a cathode field of $50 \mathrm{MV} / \mathrm{m}$, changes according to Eq. (4) from $0.27 \mathrm{eV}$ to $0.60 \mathrm{eV}$. Inserting the above numbers and a photon energy corresponding to a laser wavelength of $262 \mathrm{~nm}$ into Eqs. (1) and (2), the estimated normalized intrinsic emittance for copper varies from practically zero (taking $\phi_{w}=4.66+0.51 \mathrm{eV}$ and $\phi_{\mathrm{Sch}}=0.27 \mathrm{eV}$ ) to about $0.9 \mu \mathrm{m} / \mathrm{mm}$ (with $\phi_{w}=$ $4.66-0.51 \mathrm{eV}$ and $\phi_{\mathrm{Sch}}=0.60 \mathrm{eV}$ ). For the same laser wavelength and cesium telluride, the predicted normalized intrinsic emittance from Eqs. (1) and (3) varies between about $0.5 \mu \mathrm{m} / \mathrm{mm}$ (with $E_{g}+E_{a}=4.6 \mathrm{eV}$ and $\phi_{\text {Sch }}=$ $0.27 \mathrm{eV}$ ) to about $1.1 \mu \mathrm{m} / \mathrm{mm}$ (taking $E_{g}+E_{a}=3.5 \mathrm{eV}$ and $\phi_{\mathrm{Sch}}=0.60 \mathrm{eV}$ ). In conclusion, due to the lack of precise knowledge of the parameters to estimate the normalized intrinsic emittance, an accurate theoretical estimation for both types of material is very difficult and measurements are required to compare the performance between different photocathodes.

\section{ELECTRON SOURCE}

Electron bunches at the SwissFEL Injector Test Facility are generated in an $S$-band rf photo-injector gun operated at $10 \mathrm{~Hz}$ repetition rate. There are two gun drive lasers: one based on a Ti:sapphire chirped pulse amplification system [24] with a nominal center wavelength of $266.6 \mathrm{~nm}$ and an approximately flat-top pulse (9.9 ps FWHM) obtained by pulse stacking, and a compact turn-key Nd:YLF amplifier with a fixed wavelength of $262.0 \mathrm{~nm}$ and a Gaussian profile (6.4 ps FWHM). Two different 2.6-cells rf guns were used during the operation of the SwissFEL Injector Test Facility: one originally developed for high-current operation at the CLIC Test Facility at CERN [25], that gives a field at the cathode at the injection phase of $50 \mathrm{MV} / \mathrm{m}$, and, for the run period after April 2014, a PSI-developed rf gun capable of operating at $100 \mathrm{~Hz}$ with a field of $76 \mathrm{MV} / \mathrm{m}$ at the cathode [26]. For both rf guns the total beam energy at the gun exit is set to $7.1 \mathrm{MeV}$.

\section{CATHODE PREPARATION AND QUANTUM EFFICIENCY}

We have tested five cathodes made of pure copper, three without coating labeled $\mathrm{Cu}-3, \mathrm{Cu}-19$ and $\mathrm{Cu}-22$, 
and two coated with cesium telluride labeled $\mathrm{Cs}_{2} \mathrm{Te}-8$ and $\mathrm{Cs}_{2} \mathrm{Te}-17$. The backplane of the rf gun is designed such that cathode plugs (see Fig. 1) can be inserted. The exchange of a cathode plug is done under vacuum with an in-vacuum manipulation arm. Up to four cathodes can be stored in a loadlock system [27] mounted behind the rf photoinjector. The exchange of a cathode from the loadlock to the gun takes less than five minutes, thus enabling the characterization of different cathode materials within the same day. This is important for the comparison of cathode materials under the same environmental conditions, given by laser, rf and vacuum characteristics. If a fresh cathode plug is mounted, an rf conditioning period varying from one to several hours is required. This conditioning is needed only once and can be done in advance before storing the cathode plugs in the loadlock chamber.

The copper cathode surface is diamond turned to an average roughness of a few nanometers [28]. We apply a careful cleaning of the cathodes consisting of ultra-sound cleaning followed by an annealing cycle under vacuum at $250{ }^{\circ} \mathrm{C}$ during 10 hours. After this procedure, the cathode can be used in the gun or transferred to the $\mathrm{Cs}_{2} \mathrm{Te}$ evaporation system. The deposition of the $\mathrm{Cs}_{2} \mathrm{Te}$ layer is done by successively evaporating $15 \mathrm{~nm}$ of tellurium and $25 \mathrm{~nm}$ of cesium. This so-called blind deposition (because there is no QE monitoring during evaporation) is adapted from a CERN recipe $[29,30]$. The method is easy to implement but produces cathodes with $\mathrm{QE}$ not larger than a few percent, to be compared to the $10 \%$ QE obtained with more efficient deposition methods. The diameter of the deposited area is defined by a mask in front of the cathode during the evaporation process and was $10 \mathrm{~mm}$ for $\mathrm{Cs}_{2} \mathrm{Te}-8$ and $5 \mathrm{~mm}$ for $\mathrm{Cs}_{2} \mathrm{Te}-17$.

Figure 2 shows the QE evolution for the five cathodes studied at the SwissFEL Injector Test Facility. The QE is measured by recording the charge at a calibrated beam position monitor $2.6 \mathrm{~m}$ downstream of the rf gun. Our preparation method has proven to be effective, ensuring an initial QE for copper above $10^{-4}$ and a QE still above $7 \times 10^{-5}$ after the extraction of more than $17 \mathrm{mC}$ charge (accumulated during 100 days of operation at $10 \mathrm{~Hz}$ ) at a pressure (vacuum) level of around $10^{-9}$ mbar. For cesium

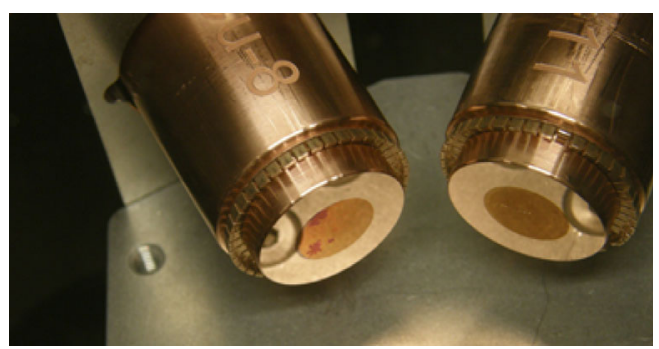

FIG. 1. SwissFEL cathode plugs parked in the carousel holder of the loadlock chamber. A disc of $10 \mathrm{~mm}$ diameter of $\mathrm{Cs}_{2} \mathrm{Te}$ has been deposited on the front surface.



FIG. 2. Quantum efficiency evolution of the five photocathodes tested at the SwissFEL Injector Test Facility. Time zero indicates the beginning of operation for each cathode individually.

telluride, our experience with $\mathrm{Cs}_{2} \mathrm{Te}-17$ has shown that a QE above $0.1 \%$ could be maintained for at least two months, during which $3 \mathrm{mC}$ were extracted, at around $10^{-9}$ mbar without any signs of continuous degradation.

\section{EMITTANCE MEASUREMENT PROCEDURE}

The emittance at the source is determined by three different contributions related to intrinsic emittance, space charge, and the rf field, respectively. When space-charge and rf-field effects are negligible, the (transverse) emittance of a single longitudinal slice of the bunch corresponds to the intrinsic emittance. In our measurements we divide the beam into ten slices per RMS bunch length and average the emittance over five slices around the longitudinal beam center to obtain the so-called core slice emittance. The error we associate with each core slice emittance measurement accounts for both the variation of the emittance along the slices and the statistical fluctuations between individual emittance measurements.

Establishing a reliable high-resolution method is crucial for the precise measurement of the electrons' slice emittance. We measure the core slice emittance by streaking the bunch and visualizing it on a high-resolution yttriumaluminum-garnet (YAG) screen in the high energy section of the accelerator. We can streak the beam either directly with an $S$-band rf transverse deflector or by introducing dispersion to an energy-chirped beam.

The YAG screen is based on a novel imaging geometry that observes the Snell-Descartes law of refraction as well as the Scheimpflug imaging condition in the scintillating crystal [31]. This allows reaching a beam size resolution much better than the crystal thickness: the scintillator is placed at an angle of 8.1 degrees with respect to the electron beam and has a thickness of $100 \mu \mathrm{m}$, while the measured resolution is $15 \mu \mathrm{m}$. 
The slice emittance is reconstructed by measuring the slice beam size for different optics transformations. The optics are varied by using five quadrupole magnets: the phase advance in the measurement direction is scanned at equidistant intervals to cover a full range of about 150 degrees, the phase advance in the streaking plane is set to optimize the longitudinal resolution, the $\beta$-function in the measurement plane is kept between $10 \mathrm{~m}$ and $50 \mathrm{~m}$ to have beam sizes that can be conveniently measured, and the $\beta$-function in the streaking direction is set to less than $10 \mathrm{~m}$ to limit the streaked beam size.

The resolution on the normalized emittance is about 2-3 nm for a final beam energy of $250 \mathrm{MeV}$. The longitudinal resolution is about $13 \mathrm{fs}$ when the transverse deflector is used. With the dispersion method we can resolve more than ten slices per RMS bunch length for an RMS energy chirp of 2\%. Assuming a 5\% beam-size measurement error, the statistical errors of the slice emittance measurement are between $2 \%$ and $3 \%$. The calibration uncertainty of the YAG screen is of the order of $1 \%$, which causes a systematic error of the reconstructed emittances of around $2 \%$. The calibration error is dominant over other systematic uncertainties such as quadrupole field errors, energy errors, or optics mismatch. Overall the errors of the slice emittance measurements are estimated to be below the 5\% level. A more detailed description of the slice emittance measurements can be found in Refs. [13,32].

The novel YAG screen has made possible reliable beamsize measurements for bunch charges as low as $1 \mathrm{pC}$ and
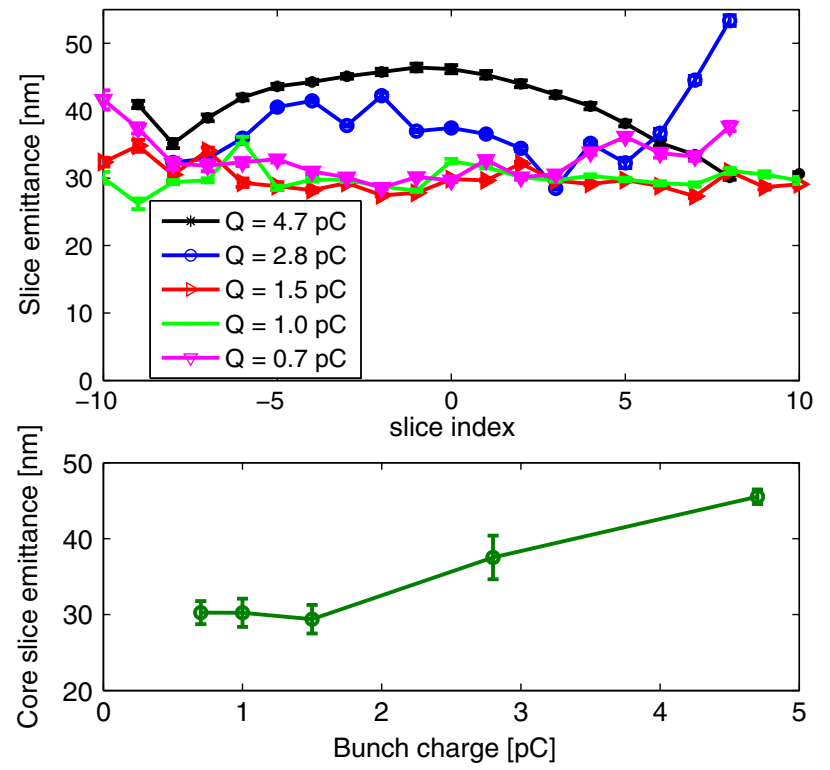

FIG. 3. Measured emittance along the bunch length (slice index) for different bunch charges $Q$ (upper plot). Core slice emittance as a function of the bunch charge (lower plot). For charges equal or below $1.5 \mathrm{pC}$ the slice emittance is not affected by space charge. below, where space-charge effects are negligible for our laser beam sizes. The threshold in surface charge density beyond which space charge becomes non-negligible is around $30 \mathrm{pC} / \mathrm{mm}^{2}$ at $50 \mathrm{MV} / \mathrm{m}$ field on the cathode with a 9.9 ps FWHM pulse length. This has been identified by reducing the bunch charge at fixed laser beam size until the emittance was no longer reduced, as shown in Fig. 3 for the cathode $\mathrm{Cu}-19$.

The normalized intrinsic emittance $\varepsilon_{\text {int }} / \sigma_{l}$ is reconstructed by measuring the core slice emittance as a function of the transverse laser beam size, which in our case is varied by changing the laser aperture. Once the space-charge limit is found, the surface charge density is kept constant during the aperture scan to avoid space charge effects. Figure 4 shows two examples of such aperture scans for the cathodes $\mathrm{Cu}-19$ and $\mathrm{Cs}_{2} \mathrm{Te}-17$. The dependence of the emittance on the laser beam size is not strictly linear as expected from Eq. (1). The deviation can be explained by our gun design, which exposes the electron beam to a quadrupole field component due to non-coaxial rf feeds from opposing sides. We have verified that the nonlinear dependence comes from the rf field by performing aperture scans at different gun gradients [33]. The effect is less pronounced in the new PSI gun. Taking the quadratic component into account the normalized intrinsic emittance is determined as the linear coefficient in a quadratic fit to the measurements. The errors of the normalized intrinsic emittances are the uncertainties of the second-order polynomial fits, which take into account the individual errors of each core slice emittance measurement. The resulting error depends on the stability of the accelerator conditions during a given aperture scan and varies between $2 \%$ and $10 \%$ for the measurements presented here.

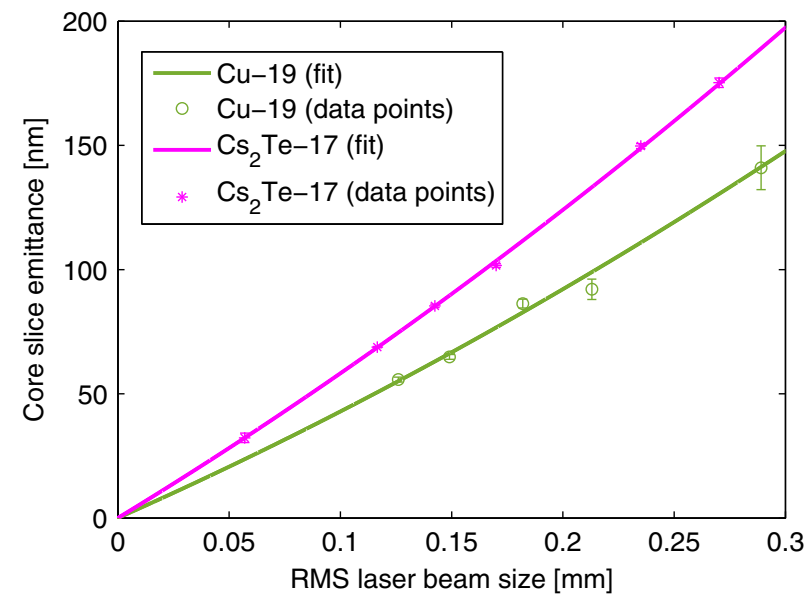

FIG. 4. Examples of normalized intrinsic emittance measurements for two of the cathodes. In the $\mathrm{Cs}_{2} \mathrm{Te}-17$ case accelerator conditions were more stable than for $\mathrm{Cu}-19$, resulting in a smaller error on the normalized intrinsic emittance. 
TABLE I. Measured normalized intrinsic emittances for the five cathodes tested at the SwissFEL Injector Test Facility.

\begin{tabular}{lccccc}
\hline \hline Cathode & Measurement day & $\begin{array}{c}\varepsilon_{\text {int }} / \sigma_{l}(\mathrm{raw}) \\
{[\mu \mathrm{m} / \mathrm{mm}]}\end{array}$ & $\begin{array}{c}\text { Laser wavelength } \\
{[\mathrm{nm}]}\end{array}$ & $\begin{array}{c}\text { Cathode field } \\
{[\mathrm{MV} / \mathrm{m}]}\end{array}$ & $\begin{array}{c}\varepsilon_{\text {int }} / \sigma_{l}(\text { normalized }) \\
{[\mu \mathrm{m} / \mathrm{mm}]}\end{array}$ \\
\hline $\mathrm{Cu}-3$ & 30 October 2012 & $0.51 \pm 0.04$ & 267.6 & 50 & $0.57 \pm 0.04$ \\
$\mathrm{Cu}-3$ & 31 October 2012 & $0.55 \pm 0.01$ & 260.1 & 50 & $0.53 \pm 0.01$ \\
$\mathrm{Cu}-19$ & 25 September 2013 & $0.44 \pm 0.02$ & 262.0 & 50 & $0.44 \pm 0.02$ \\
$\mathrm{Cu}-19$ & 04 April 2014 & $0.40 \pm 0.03$ & 262.0 & 50 & $0.40 \pm 0.03$ \\
$\mathrm{Cu}-22$ & 13 June 2014 & $0.58 \pm 0.03$ & 262.0 & 76 & $0.54 \pm 0.03$ \\
$\mathrm{Cs}_{2} \mathrm{Te}-8$ & 04 April 2014 & $0.54 \pm 0.06$ & 262.0 & 50 & $0.54 \pm 0.06$ \\
$\mathrm{Cs}_{2} \mathrm{Te}-17$ & 08 October 2014 & $0.54 \pm 0.01$ & 266.6 & 76 & $0.54 \pm 0.01$ \\
$\mathrm{Cs}_{2}$ Te-17 & 08 October 2014 & $0.50 \pm 0.02$ & 266.6 & 76 & $0.51 \pm 0.02$ \\
$\mathrm{Cs}_{2} \mathrm{Te}-17$ & 08 October 2014 & $0.52 \pm 0.02$ & 266.6 & 76 & $0.53 \pm 0.02$ \\
\hline \hline
\end{tabular}

\section{MEASUREMENT RESULTS}

Table I and Fig. 5 show the measured normalized intrinsic emittances for the five cathodes tested at the SwissFEL Injector Test Facility. The numbers listed in the last column of Table I and displayed in Fig. 5 have been normalized to a laser wavelength of $262.0 \mathrm{~nm}$ and to a cathode field of $50 \mathrm{MV} / \mathrm{m}$. The normalization to the laser wavelength is done according to Eqs. (1)-(3), and the normalization to the field at the cathode is done based on the Schottky effect [18]. The dependence of the intrinsic emittance on the laser wavelength was demonstrated in a pulsed diode gun at PSI [34]. More recently, the scalings with both the laser wavelength and the cathode field have been verified experimentally at the SwissFEL Injector Test Facility [33].

Three consecutive measurements were done for the cathode $\mathrm{Cs}_{2} \mathrm{Te}-17$. The reconstructed normalized intrinsic emittances for the three measurements were equivalent. Two of the cathodes $(\mathrm{Cu}-3$ and $\mathrm{Cu}-19)$ were measured on two different days each. The measured normalized intrinsic emittances for the same cathode do not differ

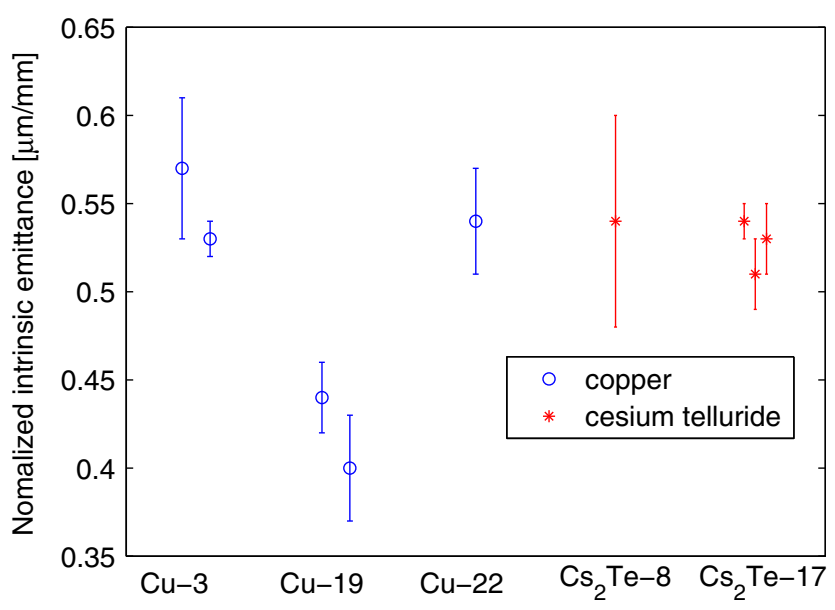

FIG. 5. Measured normalized intrinsic emittances for the five cathodes studied at the SwissFEL Injector Test Facility. significantly between measurement days, indicating that the uncertainty introduced by the overall stability of accelerator and laser system is comparable to the errors of individual measurements.

The normalized intrinsic emittance for the copper cathodes approximately ranges between $0.37 \mu \mathrm{m} / \mathrm{mm}$ and $0.61 \mu \mathrm{m} / \mathrm{mm}$. This is consistent with the expected theoretical values and with our measured $\mathrm{QE}$ at the $10^{-4}$ level [15]. For cesium telluride, the normalized intrinsic emittance varies between about $0.48 \mu \mathrm{m} / \mathrm{mm}$ and about $0.60 \mu \mathrm{m} / \mathrm{mm}$, in reasonable agreement with the theoretical expectations given in the introduction. Our measured normalized intrinsic emittances are generally smaller than the values obtained in previous studies. A notable exception is given by Ref. [35], where an indirect measurement method resulted in similar emittance values for $\mathrm{Cs}_{2} \mathrm{Te}$.

\section{DISCUSSION}

The intrinsic emittance and the quantum efficiency depend on the surface atomic composition (number of contaminants) and the surface morphology (local crystal orientation, mechanical defects), which will locally enhance more or less the applied electric field. These physical parameters may vary during beam operation due to activity on the cathode surface, such as field emission produced by some local hot spots, the deposition on the surface of vacuum contaminants that might be cracked or removed by the laser, etc. Such dynamic activity may change the cathode performance over time, as illustrated in the case of the quantum efficiency in Fig. 2.

In addition, the surface material composition and morphology depend on how the cathode was prepared. The diamond machining of the cathode surface is not exactly reproducible from one cathode to the next, leaving more or fewer mechanical defects on the sub-micrometer scale. In the same way the cathode cleaning/annealing will not always produce the same surface state: the removal of the contaminants (like water and machining grease) and the size and type of crystal grains exposed to the laser illumination may vary. These small differences between 
cathodes supposed to be identical can explain the variations in intrinsic emittance between the copper cathodes shown in Table I and Fig. 5 and the QE variations between cathodes of the same material shown in Fig. 2.

The observed variations over time and between different cathodes do not prevent to conclude that the QE is much larger for cesium telluride than for copper, while the intrinsic emittance is practically equivalent for both materials.

\section{CONCLUSION}

We have measured the intrinsic emittance for copper and cesium telluride for the first time under the same conditions in the same rf photoinjector. Cesium telluride cathodes provide a $\mathrm{QE}$ orders of magnitude higher than standard copper cathodes at only minimal loss in emittance. The excess of QE and hence laser energy can be used to improve the beam emittance by optimizing the laser beam quality. Alternatively, the photoinjector may be driven by a less powerful and thus more affordable laser system. We have measured normalized intrinsic emittances consistent with theory and down to about $0.40 \mu \mathrm{m} / \mathrm{mm}$. The experimental verification of such small values was made possible through the combination of our high-sensitivity profile monitor and our reliable high-resolution measurement procedure.

\section{ACKNOWLEDGMENTS}

We would like to thank the beam diagnostics group of PSI, in particular Rasmus Ischebeck, for developing and implementing the SwissFEL profile monitor used for our measurements. In addition we acknowledge the extensive contributions of all the PSI expert groups and the SwissFEL team to the construction and operation of the SwissFEL Injector Test Facility. We finally thank Marco Pedrozzi for careful proofreading of the manuscript.

[1] J. S. Fraser, R. L. Sheffield, E. R. Gray, and G. W. Rodenz, IEEE Trans. Nucl. Sci. 32, 1791 (1985); for a recent review see, e.g., C. Hernandez-Garcia, P. G. O' Shea, and M. L. Stutzman, Phys. Today 61, 44 (2008).

[2] S. Boucher, P. Frigola, A. Murokh, M. Ruelas, I. Jovanovic, J. B. Rosenzweig, and G. Travish, Nucl. Instrum. Methods Phys. Res., Sect. A 608, S54 (2009).

[3] W. S. Graves, W. Brown, F.X. Kaertner, and D. E. Moncton, Nucl. Instrum. Methods Phys. Res., Sect. A 608, S103 (2009).

[4] J. B. Hastings, F. M. Rudakov, D. H. Dowell, J. F. Schmerge, J. D. Cardoza, J. M. Castro, S. M. Gierman, H. Loos, and P. M. Weber, Appl. Phys. Lett. 89, 184109 (2006).

[5] P. Musumeci, J. T. Moody, and C. M. Scoby, Ultramicroscopy 108, 1450 (2008).

[6] R. Ganter, PSI Report No. 10-04, 2012.
[7] O. J. Luiten, S. B. van der Geer, M. J. de Loos, F. B. Kiewiet, and M. J. van der Wiel, Phys. Rev. Lett. 93, 094802 (2004).

[8] F. Zhou, A. Brachmann, P. Emma, S. Gilevich, and Z. Huang, Phys. Rev. ST Accel. Beams 15, 090701 (2012).

[9] H. J. Qian, C. Li, Y. C. Du, L. X. Yan, J. F. Hua, W. H. Huang, and C.X. Tang, Phys. Rev. ST Accel. Beams 15, 040102 (2012).

[10] Y. Ding et al., Phys. Rev. Lett. 102, 254801 (2009).

[11] F. Stephan et al., Phys. Rev. ST Accel. Beams 13, 020704 (2010).

[12] M. Pedrozzi, PSI Report No. 10-05, 2010.

[13] E. Prat, M. Aiba, S. Bettoni, B. Beutner, S. Reiche, and T. Schietinger, Phys. Rev. ST Accel. Beams 17, 104401 (2014).

[14] S. Bettoni et al. (to be published).

[15] D. H. Dowell and J. F. Schmerge, Phys. Rev. ST Accel. Beams 12, 074201 (2009).

[16] K. Flöttmann, TESLA FEL Report No. 1997-01, 1997.

[17] D. H. Dowell, I. Bazarov, B. Dunham, K. Harkay, C. Hernandez-Garcia, R. Legg, H. Padmore, T. Rao, J. Smedley, and W. Wan, Nucl. Instrum. Methods Phys. Res., Sect. A 622, 685 (2010).

[18] Z. M. Yusof, M. E. Conde, and W. Gai, Phys. Rev. Lett. 93, 114801 (2004).

[19] D. H. Dowell, F. K. King, R. E. Kirby, J. F. Schmerge, and J. M. Smedley, Phys. Rev. ST Accel. Beams 9, 063502 (2006).

[20] R. A. Powell, W. E. Spicer, G. B. Fisher, and P. Gregory, Phys. Rev. B 8, 3987 (1973).

[21] E. Chevallay, S. Hutchins, P. Legros, G. Suberlucq, and H. Trautner, in Proceedings of the 20th International Linac Conference, Monterey, USA, 2000 (SLAC, Menlo Park, 2000), p. 110.

[22] H. Sugiyama, K. Ogawa, J. Azuma, K. Takahashi, and M. Kamada, AIP Conf. Proc. 1149, 1067 (2009).

[23] H. Chen, Y. Du, W. Gai, A. Grudiev, J. Hua, W. Huang, J. G. Power, E. E. Wisniewski, W. Wuensch, C. Tang, L. Yan, and Y. You, Phys. Rev. Lett. 109, 204802 (2012).

[24] A. Trisorio, P. M. Paul, F. Ple, C. Ruchert, C. Vicario, and C. P. Hauri, Opt. Express 19, 20128 (2011).

[25] R. Bossart and M. Dehler, in Proceedings of the 5th European Particle Accelerator Conference, Sitges, Spain, 1996 (IOP, Bristol, 1996), p. 1544.

[26] J.-Y. Raguin, M. Bopp, A. Citterio, and A. Scherer, in Proceedings of the 26th International Linear Accelerator Conference, Tel Aviv, Israel, 2012 (JACoW, Geneva, 2012), p. 495.

[27] R. Ganter et al., in Proceedings of the 35th International Free-Electron Laser Conference, New York, USA, 2013 (JACoW, Geneva, 2013), p. 259.

[28] J. Bossert, R. Ganter, M. Schaer, and T. Schietinger, in Proceedings of the 36th International Free-Electron Laser Conference, Basel, Switzerland, 2014 (JACoW, Geneva, 2015), p. 832.

[29] E. Chevallay (private communication).

[30] E. Chevallay, J. Durand, S. Hutchins, G. Suberlucq, and H. Trautner, in Proceedings of the 19th International 
Linear Accelerator Conference, Chicago, USA, 1998 (Argonne National Laboratory, Argonne, 1998), p. 872.

[31] R. Ischebeck and V. Thominet, Patent WO2014029693A1, 2014.

[32] E. Prat and M. Aiba, Phys. Rev. ST Accel. Beams 17, 032801 (2014).

[33] E. Prat, S. Bettoni, H. H. Braun, M. C. Divall, R. Ganter, C. P. Hauri, T. Schietinger, A. Trisorio, and C. Vicario, in Proceedings of the 36th International
Free Electron Laser Conference, Basel, Switzerland, 2014 (JACoW, Geneva, 2015), p. 970.

[34] C.P. Hauri, R. Ganter, F. Le Pimpec, A. Trisorio, C. Ruchert, and H.H. Braun, Phys. Rev. Lett. 104, 234802 (2010).

[35] D. Sertore, D. Favia, P. Michelato, L. Monaco, and P. Pierini, in Proceedings of the 9th European Particle Accelerator Conference, Lucerne, Switzerland, 2004 (EPS-AG, Mulhouse, 2004), p. 408. 